\title{
Effets de l'introgression dans le génome Piétrain de l'allèle normal au locus de sensibilité à l'halothane
}

\author{
R Hanset, C Dasnois, S Scalais, C Michaux, L Grobet \\ Service de génétique factorielle et moléculaire, faculté de médecine vétérinaire \\ de l'université de Liège, 20, bd de Colonster, B 43, B-4000 Liège, Belgique
}

(Reçu le 27 octobre 1993; accepté le 28 septembre 1994)

\begin{abstract}
Résumé - Dans cet article, on décrit les étapes de la reconstruction du génome Piétrain par backcross successifs (BC1 3/4; BC2 7/8; BC3 15/16) tout en maintenant l'hétérozygotie au locus $\mathrm{Hal}$ chez les truies reproductrices. Les informations récoltées concernent : la croissance, la conformation au sevrage et à la fin de l'engraissement, des mensurations prises sur la carcasse, la composition de la carcasse, des critères de qualité de viande (mesures du $\mathrm{pH}$ et de la réflectance par le FOP). En tout, 30 critères sont envisagés. Ils se rapportent à 1890 porcs et ont été analysés selon un modèle comportant comme effets : génotype au locus $\mathrm{Hal}$, numéro de backcross, interaction "génotype $\times$ backcross ", sexe, mois-année, et avec le poids d'abattage comme covariable. La différence entre les génotypes $n n$ et $N n$ n'était influencée par le numéro de backcross que pour les $\mathrm{pH} 1 \mathrm{du}$ $\mathrm{m}$ Longissimus dorsi (carré) et du $\mathrm{m}$ Gracilis (jambon). Par rapport au génotype $N n$, le génotype $n n$ est plus court, a un meilleur rendement à l'abattage, est plus charnu, plus compact et par conséquent mieux conformé, a des mesures du $\mathrm{pH} 1$ plus basses et des mesures des FOP1 et FOP24 plus élevées. Avec la progression des backcross et quel que soit le génotype au locus $\mathrm{Hal}$, les carcasses deviennent plus courtes, plus riches en viande et mieux conformées. D'ailleurs, la composition de la carcasse et le classement commercial des carcasses des sujets de génotype $n n$ au stade $15 / 16$ sont quasi identiques à ceux du Piétrain pur. L'éventualité d'une exploitation du gène $n$ à l'état hétérozygote mais dans un contexte Piétrain reconstitué est discutée.
\end{abstract}

Piétrain / locus $\mathrm{Hal} /$ backcross / introgression

Summary - Introgression into the Piétrain genome of the normal allele at the locus for halothane sensitivity. This paper describes the steps of reconstruction of the Piétrain genome by repeated backcrosses (BC1 3/4; BC2 7/8;BC3 15/16) while maintaining heterozygosis at the Hal locus in breeding females. The following traits were recorded: growth, conformation at weaning and at the end of the fattening period, measurements taken on the carcass, carcass composition, meat quality criteria ( $p H$ and reflectance (FOP) measurements). In all, 30 criteria were considered and 1890 pigs were involved in this study. The statistical model of analysis included the following terms: genotype at the Hal 
locus; backcross number; 'genotype $\times$ backcross number' interaction; sex; year-month; and slaughter weight as a covariate. The difference between the $\mathrm{nn}$ and $\mathrm{Nn}$ genotypes was not influenced by the backcross number except for the pH1 measurements of the $m$ Longissimus dorsi and Gracilis. Compared to the Nn genotype, the nn genotype is shorter, has a better killing-out-percentage, is more meaty and more compact and has a better conformation, lower pH1 measurements and higher FOP1 and FOP24 values. With the progress of the experiment and whatever the genotype at the Hal locus, the carcass became shorter and leaner. The carcass composition and the commercial classification of the animals of the nn genotype have reached the 15/16 level and are practically identical to those of the pure Piétrain. Possible ways of exploiting the $\mathrm{n}$ gene in the heterozygous state but within a reconstructed Piétrain background are discussed.

Piétrain pig / halothane locus / backcross introgression

\section{INTRODUCTION}

L'entité biologique qu'est le Piétrain est déterminée par l'homozygotie nn au locus $\mathrm{Hal}$ ainsi que par d'autres gènes, non encore identifiés, dont l'action globale sur la composition corporelle est analogue à celle du gène $n$ (Hanset et al 1995).

En outre, si l'allèle $n$ est récessif pour la sensibilité à l'halothane, en revanche, il s'exprime déjà chez l'hétérozygote pour la plupart des caractères zootechniques envisagés et en particulier pour la composition de la carcasse et la conformation qu'il influence favorablement. Dès lors, outre la combinaison génétique «génotype $n n+$ autres gènes Piétrain» caractérisant le porc de Piétrain classique, on peut envisager d'autres combinaisons telles que : "génotype $N n$ ou $N N$ + autres gènes Piétrain ". Ces dernières font donc appel au gène $N$ qui doit être introduit dans le contexte génétique Piétrain par un programme d'introgression. Un tel programme consiste à transférer un gène d'une race donneuse dans une autre race dite récurrente, laquelle ne diffère en fin de compte de sa version originale que par le seul gène transféré ou par un court segment chromosomique contenant ce gène.

Pareille expérience a été entreprise dans le but d'introduire le gène $N$ de résistance à l'halothane hébergé par le Large White (race donneuse) dans une souche Piétrain (race récurrente).

La première partie d'un tel travail consiste à reconstituer autour de l'hétérozygotie $N n$, le génome de la race Piétrain par backcross répétés, l'étape ultérieure étant constituée par l'intercross $N n \times N n$ de manière à produire des sujets $N N$, homozygotes résistants. Chaque backcross comporte le croisement de truies hétérozygotes $N n$ avec des verrats Piétrain $n n$. Sont ainsi produits $50 \%$ d'homozygotes $n n$ et $50 \%$ d'hétérozygotes $N n$. Parmi les femelles $N n$, on choisit des truies destinées à engendrer le backcross suivant. Au départ, les génotypes au locus $\mathrm{Hal}$ étaient identifiés par le test à l'halothane combiné au typage pour un marqueur biochimique étroitement lié, la phosphohexose isomérase. Depuis la découverte par Fuji et al (1991) de la mutation ponctuelle $(\mathrm{C} \rightarrow \mathrm{T})$ en position 1843 de l'ADNc du gène codant pour le récepteur membranaire à la ryanodine et responsable de la sensibilité à l'halothane, il est désormais possible de déterminer le génotype au locus $\mathrm{Hal}$ de tout individu par un test génomique direct basé sur la présence ou l'absence de la mutation. 
Cet article décrit l'évolution des performances zootechniques des 2 génotypes $N n$ et $n n$ produits lors des backcross successifs : backcross $1(3 / 4)$, backcross $2(7 / 8)$, backcross $3(15 / 16)$ et étudie l'influence du contexte génétique en cours de reconstitution sur la différence entre les 2 génotypes.

\section{MATÉRIEL ET MÉTHODES}

Cette expérience fait suite à la production d'une F2 obtenue au départ d'un croisement Piétrain $\times$ Large White et décrite par Hanset et al (1995). Les truies étaient de génotype $B n / A N$, c'est-à-dire portant sur le chromosome 6 d'origine paternelle l'haplotype $B n$ ( $n$, allèle de la sensibilité à l'halothane, locus $H a l$; $B$, allèle au locus $P H I$ de la phosphohexose-isomérase, locus étroitement lié au précédent) et sur le chromosome homologue d'origine maternelle, l'haplotype $A N$ $(N$, allèle au locus $H a l$ et $A$, allèle au locus $P H I)$. Ces truies $\mathrm{F} 1$, après avoir servi à produire la F2, furent utilisées comme point de départ d'une série de backcross à des verrats Piétrain de génotype $B n / B n$. La combinaison du typage pour la $P H I$ et du test à l'halothane devait garantir, avec un haut degré de sécurité, l'identification des génotypes et en particulier l'hétérozygotie des femelles sélectionnées pour engendrer le backcross suivant. Lorsque le test génomique direct fut adopté fin 1991, en remplacement des tests précédents, il s'est avéré que les mères des backcross étaient bien hétérozygotes au locus $\mathrm{Hal}$ (Grobet et al, 1992; Grobet, 1993).

Les conditions expérimentales et les critères zootechniques mesurés ont été décrits dans l'article précédent consacré à la F2 du croisement Piétrain $\times$ Large White (Hanset et al, 1995). Toutefois, dans le courant du backcross $2(7 / 8)$, des critères supplémentaires de qualité de viande ont été introduits. Ainsi, outre les $\mathrm{pH} 1$ et $\mathrm{pH} 24$ mesurés au niveau du m Longissimus dorsi (carré), du m Gracilis (jambon 1), les mêmes mesures ont été effectuées au niveau du m Semimembranosus (jambon 2). De plus, des mesures de réflectance ont été prises à l'aide d'une sonde «Fiber Optic Probe» (FOP) de la firme Tecpro. Cet appareil est calibré de manière telle que la viande «normale» donne des valeurs entre 25 et 50 , la viande PSE des valeurs entre 55 et 80 , la viande «DFD» des valeurs inférieures à 20 . Les mesures ont été faites après $1 \mathrm{~h}$ (FOP1) et $24 \mathrm{~h}$ (FOP24) aux mêmes endroits que les mesures du pH (carré, jambon 1, jambon 2).

Le backcross 1 (3/4) s'est déroulé d'août 1989 à mars 1991 et est issu de 48 truies et 26 verrats, le backcross $2(7 / 8)$ d'octobre 1990 à novembre 1992 et est issu de 69 truies et 34 verrats, le backcross 3 (15/16) d'avril 1992 à décembre 1993 et est issu de 60 truies et 21 verrats. Tous ces verrats appartenaient au Centre provincial d'insémination artificielle d'Argenteau (Liège). Comme il y avait chevauchement dans le déroulement des 3 backcross, ceux-ci ont pu faire l'objet d'une analyse unique. Au total, 1890 sujets sont concernés par cette étude.

Le modèle statistique d'analyse comporte les effets suivants : le génotype au locus Hal (2 niveaux), le numéro de backcross ( 3 niveaux), l'interaction "génotype $\times$ backcross » $(2 \times 3$ niveaux $)$, le sexe ( 2 niveaux), l'année-mois d'abattage ( 54 niveaux) et, en outre, le poids d'abattage comme covariable. Le nombre de porcs abattus chaque semaine étant fort variable, pour l'analyse on a préféré rassembler les abattages d'un même mois. On a pu confirmer que, dans nos conditions d'abattage, le «mois d'abattage» pouvait tenir lieu de «jour d'abattage» sans affecter de 
manière sensible les résultats concernant les critères de qualité de viande. Cette analyse statistique a été réalisée à l'aide de la procédure GLM de SAS (1985).

\section{RÉSULTATS ET DISCUSSION}

Les niveaux de signification des effets sont donnés au tableau I, tandis que les moyennes des moindres carrés avec erreurs standard correspondant aux effets «interaction » et «sexe» sont donnés aux tableau II pour les caractères de croissance et de composition de carcasse, au tableau III pour les critères de qualité de viande. Son concernés : i) 18 caractères en relation avec la croissance, la conformation, la composition de carcasse, ii) 12 critères en rapport avec la qualité de la viande.

Le terme «interaction backcross $\times$ génotype» est généralement non-significatif, ce qui implique que la différence entre les génotypes $N n$ et $n n$ est indépendante du niveau $(3 / 4,7 / 8,15 / 16)$ de reconstitution du génome Piétrain. C'est notamment le cas pour le gain quotidien, la longueur de la carcasse (fig 1), les mesures du gras dorsal, le pourcentage de morceaux maigres (fig 2), les indices de compacité, le $\mathrm{pH} 1 \mathrm{du}$ jambon 2, les $\mathrm{pH} 24$ et les mesures de réflectance. En revanche, il existe une interaction significative pour : i) l'épaisseur du gras dorsal au niveau du cou, la note de conformation au sevrage et la note de conformation finale, soit que, au backcross 3 , la différence entre les génotypes se soit réduite ou ait disparu (tableau II) ; manifestement, la discrimination entre génotypes en ce qui concerne la conformation (note subjective) devient plus difficile à ce stade de reconstitution du génome, déjà très proche du Piétrain pur; ii) les $\mathrm{pH} 1 \mathrm{du}$ carré et du jambon 1 , la différence entre génotypes devenant plus importante avec le numéro du backcross (tableau III). La différence entre les génotypes $N n$ et $n n$ pour le $\mathrm{pH} 1 \mathrm{du}$ carré est nettement plus importante ici que dans le F2 analysée par Hanset et al (1995). Nous ne nous expliquons pas cette observation. Il faut toutefois noter que, dans les backcross 2 et 3, on rejoint les différences observées par Barton-Gade (1981) et Wittmann et al (1993).

Le génotype au locus $\mathrm{Hal}$ exerce un effet hautement significatif sur les caractères envisagés, à l'exception du gain quotidien (malgré une tendance à la supériorité de l'hétérozygote), des pH24 du carré et du jambon 2. Les pH1 du carré et du jambon 2 sont les plus influencés par le génotype. En fait, ces 2 muscles sont des muscles «blancs» dont le métabolisme est surtout du type anaérobique (BartonGade, 1981).

D'autre part, le pH24 du jambon 1 est légèrement plus élevé chez le génotype $n n$, ce qui avait déjà été observé au niveau de la F2 (Hanset et al, 1995). Les différences entre génotypes pour les mesures du pH1 tendent à s'estomper $24 \mathrm{~h}$ après l'abattage. Ceci a également été observé par Jensen et Barton-Gade (1985), Wittman et al (1993). En revanche, en ce qui concerne les mesures de réflectance, la différence entre génotypes est toujours présente $24 \mathrm{~h}$ après l'abattage. À ce propos, il est utile de mentionner que Eikelenboom et Nanni Costa (1988) ont aussi trouvé des valeurs de FOP plus hautes chez les sujets halothane positifs et que ces valeurs s'élèvent lors des mesures ultérieures. 
Tableau I. Niveaux de signification des effets du modèle d'analyse.

\begin{tabular}{|c|c|c|c|c|}
\hline & $\begin{array}{l}\text { Numéro de } \\
\text { backcross }\end{array}$ & $\begin{array}{l}\text { Génotype au } \\
\text { locus Hal }\end{array}$ & $\begin{array}{c}\text { Interaction } n^{\circ} \text { de } \\
\text { backcross } \times \text { génotype }\end{array}$ & Sexe \\
\hline Gain quotidien (kg) & NS & NS & NS & $* * *$ \\
\hline $\begin{array}{l}\text { Longueur de } \\
\text { carcasse }(\mathrm{cm})\end{array}$ & $* * *$ & $* * *$ & + & $* * *$ \\
\hline $\begin{array}{l}\text { Épaisseur du gras } \\
\text { dorsal (cou) }(\mathrm{cm})\end{array}$ & $*$ & $* * *$ & $*$ & $* * *$ \\
\hline $\begin{array}{l}\text { Épaisseur du gras } \\
\text { dorsal }\left(1^{\text {re }} \text { vertèbre }\right. \\
\text { lombaire })(\mathrm{cm})\end{array}$ & NS & $* *$ & NS & $* * *$ \\
\hline $\begin{array}{l}\text { Épaisseur du gras } \\
\text { dorsal (croupe) }(\mathrm{cm})\end{array}$ & $* * *$ & $* * *$ & NS & $* * *$ \\
\hline $\begin{array}{l}\text { Épaisseur moyenne } \\
\text { du gras dorsal }(\mathrm{cm})\end{array}$ & $* * *$ & $* * *$ & NS & $* * *$ \\
\hline Rendement (\%) & NS & $* * *$ & NS & $*$ \\
\hline Carré (\%) & $* * *$ & $* * *$ & NS & $* * *$ \\
\hline Jambon (\%) & $* *$ & $* * *$ & NS & $* * *$ \\
\hline Épaule (\%) & NS & $* * *$ & NS & $* * *$ \\
\hline $\begin{array}{l}\text { Morceaux } \\
\text { maigres (\%) }\end{array}$ & $* * *$ & $* * *$ & NS & $* * *$ \\
\hline Bardière (\%) & $* * *$ & $* * *$ & NS & $* * *$ \\
\hline Paroi ventrale (\%) & $* * *$ & $* * *$ & NS & $* * *$ \\
\hline Morceaux gras (\%) & $* * *$ & $* * *$ & NS & $* * *$ \\
\hline $\begin{array}{l}\text { Conformation au } \\
\text { sevrage (0 à 10) }\end{array}$ & $*$ & $* * *$ & $* * *$ & NS \\
\hline $\begin{array}{l}\text { Conformation finale } \\
(0 \text { à } 10)\end{array}$ & $* * *$ & $* * *$ & $*$ & $* * *$ \\
\hline Indice de compacité 1 & $* *$ & $* * *$ & NS & $* * *$ \\
\hline Indice de compacité 2 & $* * *$ & $* * *$ & NS & $* * *$ \\
\hline $\begin{array}{l}\text { pH1 carré } \\
\text { (m Longissimus dorsi) }\end{array}$ & NS & $* * *$ & $* * *$ & $\mathrm{NS}$ \\
\hline $\begin{array}{l}\mathrm{pH} 1 \text { jambon } 1 \\
(\mathrm{~m} \text { Gracilis })\end{array}$ & NS & $* * *$ & $* * *$ & $* * *$ \\
\hline $\begin{array}{l}\mathrm{pH} 1 \text { jambon } 2 \\
\quad(\mathrm{~m} \text { Semi-membranosus })\end{array}$ & NS & $* * *$ & NS & NS \\
\hline $\begin{array}{l}\text { pH24 carré } \\
\text { (m Longissimus dorsi }\end{array}$ & $*$ & + & NS & $* * *$ \\
\hline $\begin{array}{l}\mathrm{pH} 24 \text { jambon } 1 \\
(\mathrm{~m} \text { Gracilis })\end{array}$ & NS & $* * *$ & NS & $* * *$ \\
\hline $\begin{array}{l}\text { pH24 jambon } 2 \\
(\mathrm{~m} \text { Semi-membranosus })\end{array}$ & NS & NS & NS & $*$ \\
\hline $\begin{array}{l}\text { FOP1 carré } \\
\quad(\mathrm{m} \text { Longissimus dorsi) }\end{array}$ & NS & $* * *$ & NS & NS \\
\hline $\begin{array}{l}\text { FOP1 jambon } 1 \\
(\mathrm{~m} \text { Gracilis })\end{array}$ & NS & $* * *$ & NS & NS \\
\hline $\begin{array}{l}\text { FOP1 jambon } 2 \\
\quad(\mathrm{~m} \text { Semi-membranosus })\end{array}$ & + & $* * *$ & NS & NS \\
\hline
\end{tabular}


Tableau I. (suite)

\begin{tabular}{lcccc}
\hline & $\begin{array}{c}\text { Numéro de } \\
\text { backcross }\end{array}$ & $\begin{array}{c}\text { Génotype au } \\
\text { locus Hal }\end{array}$ & $\begin{array}{c}\text { Interaction } n^{\circ} \text { de } \\
\text { backcross } \times \text { génotype }\end{array}$ & Sexe \\
\hline $\begin{array}{l}\text { FOP24 carré } \\
(\mathrm{m} \text { Longissimus dorsi) }\end{array}$ & $*$ & $* * *$ & $\mathrm{NS}$ & $\mathrm{NS}$ \\
$\begin{array}{c}\text { FOP24 jambon 1 } \\
(\mathrm{m} \text { Gracilis })\end{array}$ & $\mathrm{NS}$ & $* * *$ & $\mathrm{NS}$ & $* * *$ \\
$\begin{array}{l}\text { FOP24 jambon 2 } \\
(\mathrm{m} \text { Semi-membranosus })\end{array}$ & $\mathrm{NS}$ & $* * *$ & $\mathrm{NS}$ & $\mathrm{NS}$ \\
\hline
\end{tabular}

Niveaux de signification : NS : $P<0,10 ;+: P<0,10 ;^{*}: P<0,05 ;{ }^{* *}: P<0,01$; *** : $P<0,001$

Dès lors, on constate que, par rapport au génotype $N n$, le génotype $n n$ est plus court, a un meilleur rendement à l'abattage, est plus charnu, plus compact et par conséquent mieux conformé, à des mesures du pH1 plus basses, des mesures du FOP1 et FOP24 plus élevées. Ces observations ne sont pas nouvelles et ont été faites par de nombreux auteurs et entre autres par Eikelenboom et al (1978), Monin et al (1980), Jensen (1981), Lampo (1981), Jensen et Barton-Gade (1985), Simpson et Webb (1989), Zhang et al (1992), Wittmann et al (1993), Hanset et al (1995).

Le numéro de backcross est sans influence sur le gain journalier, en revanche, il a un effet significatif sur tous les critères en relation avec la composition de la carcasse et la conformation. Font exception l'épaisseur du gras dorsal ( $1^{\text {re }}$ vertèbre lombaire), le rendement à l'abattage, le pourcentage d'épaule (tableau I). En d'autres termes, comme on peut le constater au tableau II, quel que soit le génotype au locus $H a l$, avec la progression des backcross, les carcasses deviennent plus courtes, plus charnues, moins grasses, plus compactes. Ceci résulte de la récupération progressive de ces «autres gènes Piétrain» dont il a été question plus haut, les génotypes au locus $\mathrm{Hal}$ étant constants. En fait, par les backcross successifs, on puise ces gènes, présents chez le Piétrain, qui sont responsables de la différence entre les Piétrain purs et les F2 de génotype $n n$ (Hanset et al, 1995).

En revanche, en ce qui concerne les critères de qualité de viande, le numéro du backcross est sans effet significatif, à l'exception du pH24 et du FOP24 du carré. Ceci signifie que, si les «autres gènes Piétrain» ont une action globale analogue à celle du gène $n$ en ce qui concerne la composition de la carcasse, il n'en est pas de même pour les critères de qualité de viande envisagés ici.

L'influence du sexe est significative pour la plupart des critères de croissance, de composition de carcasse. Pour cette catégorie de caractères, seule fait exception la note de conformation au sevrage. Le gain journalier est plus élevé chez le castrat, en revanche, chez la femelle, la carcasse est plus longue et plus riche en morceaux maigres, la note de conformation finale et l'indice de compacité 2 sont plus élevés. Ces observations avaient déjà été faites sur la F2 (Hanset et al, 1995). Quant aux mesures de qualité de viande, on constate que les mesures de $\mathrm{pH}$ ont tendance à être légèrement plus élevées chez les castrats, alors que c'est l'inverse pour les mesures de réflectance. 
Table II. Moyennes des moindres carrés ( \pm erreurs standard) par backcross, génotype et sexe. Croissance et composition de carcasse.

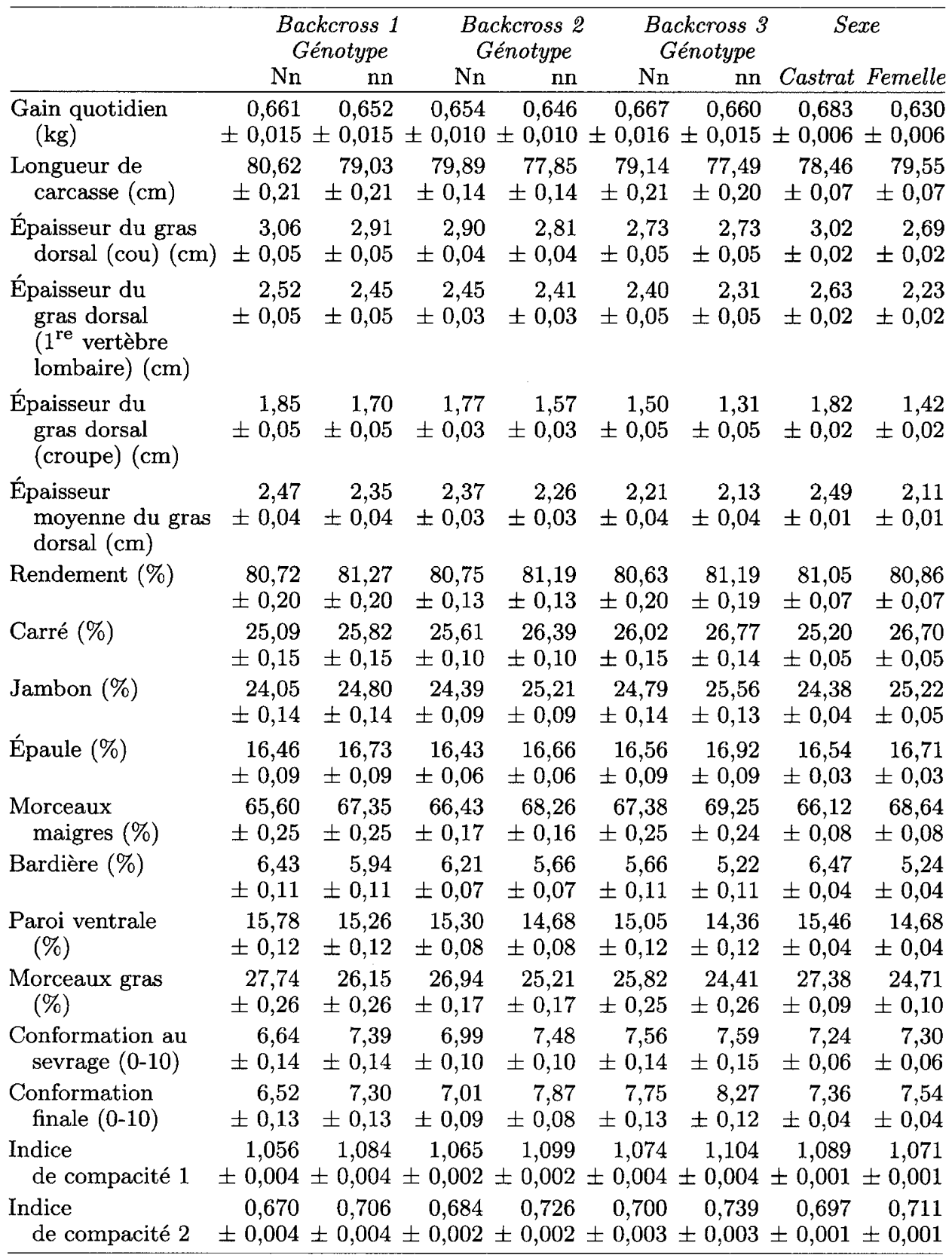

Les effectifs varient de 185 à 379 selon la combinaison backcross-génotype 
Il n'est pas sans intérêt de connaître la manière dont le marché perçoit la qualité «boucherie» de ces backcross. Le tableau IV et la figure 3 répondent à cette question. Le tableau IV donne, en outre, pour comparaison, le classement commercial de 273 porcs Piétrain purs élevés dans les mêmes conditions. On peut constater l'effet marqué du génotype, du sexe et du numéro de backcross sur le pourcentage d'animaux figurant dans les classes supérieures $(\mathrm{AA}+\mathrm{E}+\mathrm{EE})$. On observe la quasi-similitude entre les sujets 15/16 de génotype $n n$ et les Piétrain purs également de génotype $n n$ avec $44,26 \%$ de castrats $15 / 16 n n$ dans les classe $A A$ et plus contre $44,96 \%$ chez le Piétrain pur et $83,6 \%$ de truies $15 / 16 n n$ contre $88,20 \%$ chez le Piétrain pur.

Table III. Moyennes des moindres carrés ( \pm erreurs standard) par backcross, génotype et sexe. Caractéristiques de la viande.

\begin{tabular}{|c|c|c|c|c|c|c|}
\hline & \multicolumn{2}{|c|}{$\begin{array}{l}\text { Backcross } 1 \\
\text { Génotype }\end{array}$} & \multicolumn{2}{|c|}{$\begin{array}{l}\text { Backcross } 2 \\
\text { Génotype }\end{array}$} & $\begin{array}{c}\text { Backcross } 3 \\
\text { Génotype }\end{array}$ & Sexe \\
\hline & $\mathrm{Nn}$ & $\mathrm{nn}$ & $\mathrm{Nn}$ & $\mathrm{nn}$ & $\mathrm{Nn}$ & Castrat Truie \\
\hline $\begin{array}{l}\text { pH1 carré } \\
\text { (m Longissimus dorsi) }\end{array}$ & $\begin{aligned} & 5,96 \\
\pm & 0,04 \pm\end{aligned}$ & $\begin{aligned} & 5,80 \\
= & 0,04 \pm\end{aligned}$ & $\begin{array}{r}6,16 \\
+0,03\end{array}$ & $\begin{aligned} & 5,64 \\
\pm & 0,03 \pm\end{aligned}$ & $\begin{array}{rr} & 6,125,65 \\
= & 0,04 \pm 0,04\end{array}$ & $\begin{array}{r}5,895,88 \\
\pm 0,01 \pm 0,01\end{array}$ \\
\hline $\begin{array}{l}\mathrm{pH} 1 \text { jambon } 1 \\
\quad(\mathrm{~m} \text { Gracilis })\end{array}$ & $\begin{array}{l}6,09 \\
\pm 0,03 \pm\end{array}$ & $\begin{aligned} & 6,07 \\
= & 0,03 \pm\end{aligned}$ & $\begin{array}{r}6,22 \\
+0,02\end{array}$ & $\begin{array}{l}6,05 \\
\pm 0,02 \pm\end{array}$ & $\begin{array}{rr} & 6,24 \quad 6,04 \\
= & 0,04 \pm 0,04\end{array}$ & $\begin{array}{rr} & 6,14 \quad 6,10 \\
\pm 0,01 \pm 0,01\end{array}$ \\
\hline $\begin{array}{l}\text { pH1 jambon } 2 \\
\quad(\mathrm{~m} \text { Semi-membranosus })\end{array}$ & & & $\begin{array}{r}6,43 \\
+0,06\end{array}$ & $\begin{array}{l}5,98 \\
\pm 0,05 \pm\end{array}$ & $\begin{array}{rr} & 6,31 \quad 5,94 \\
= & 0,04 \pm 0,04\end{array}$ & $\begin{array}{rr}6,19 & 6,14 \\
\pm & 0,03 \pm 0,03\end{array}$ \\
\hline $\begin{array}{l}\mathrm{pH} 24 \text { carré } \\
\quad(\mathrm{m} \text { Longissimus dorsi) }\end{array}$ & $\begin{aligned} & 5,67 \\
\pm & 0,02 \pm\end{aligned}$ & $\begin{aligned} & 5,67 \\
= & 0,02 \pm\end{aligned}$ & $\begin{array}{r}5,72 \\
\pm 0,02\end{array}$ & $\begin{array}{l}5,70 \\
\pm 0,02 \pm\end{array}$ & $\begin{array}{rr} & 5,80 \quad 5,76 \\
= & 0,03 \pm 0,03\end{array}$ & $\begin{array}{rr}5,73 & 5,71 \\
\pm 0,01 & \pm 0,01\end{array}$ \\
\hline $\begin{array}{l}\mathrm{pH} 24 \text { jambon } 1 \\
\quad(\mathrm{~m} \text { Gracilis })\end{array}$ & $\begin{array}{r}6,04 \\
\pm 0,03 \pm\end{array}$ & $\begin{aligned} & 6,12 \\
= & 0,03 \pm\end{aligned}$ & $\begin{array}{r}6,07 \\
\pm 0,02\end{array}$ & $\begin{array}{l}6,14 \\
\pm 0,02 \pm\end{array}$ & $\begin{array}{rr} & 6,03 \quad 6,07 \\
= & 0,03 \pm 0,03\end{array}$ & $\begin{array}{rr}6,11 & 6,04 \\
\pm & 0,01 \pm 0,01\end{array}$ \\
\hline $\begin{array}{l}\text { pH24 jambon } 2 \\
\quad(\mathrm{~m} \text { Semi-membranosus })\end{array}$ & & & $\begin{array}{r}5,93 \\
+0,03\end{array}$ & $\begin{array}{l}5,96 \\
\pm 0,03 \pm\end{array}$ & $\begin{array}{rr}5,91 & 5,94 \\
= & 0,03 \pm 0,03\end{array}$ & $\begin{array}{rr}5,96 & 5,91 \\
\pm 0,02 \pm 0,02\end{array}$ \\
\hline $\begin{array}{l}\text { FOP1 carré } \\
\quad(\mathrm{m} \text { Longissimus dorsi) }\end{array}$ & & & $\begin{array}{r}16,35 \\
\pm 2,10\end{array}$ & $\begin{array}{r}27,78 \\
\pm 1,192 \pm\end{array}$ & $\begin{array}{r}11,84 \quad 25,99 \\
=1,37 \pm 1,27\end{array}$ & $\begin{array}{r}19,77 \quad 21,22 \\
\pm 1,07 \pm 1,08\end{array}$ \\
\hline $\begin{array}{l}\text { FOP1 jambon } 1 \\
\text { (m Gracilis) }\end{array}$ & & & $\begin{array}{r}19,04 \\
\pm 1,33\end{array}$ & $\begin{array}{r}23,26 \\
\pm 1,22 \pm\end{array}$ & $\begin{array}{r}20,52 \quad 25,12 \\
=0,87 \pm 0,81\end{array}$ & $\begin{array}{rr}21,47 & 22,51 \\
\pm 0,68 \pm 0,68\end{array}$ \\
\hline $\begin{array}{l}\text { FOP1 jambon } \\
\quad(\mathrm{m} \text { Semi-membranosus })\end{array}$ & & & $\begin{array}{r}29,12 \\
\pm 1,66\end{array}$ & $\begin{array}{r}31,21 \\
\pm 1,52 \pm\end{array}$ & $\begin{array}{r}25,3329,03 \\
=1,01 \pm 1,08\end{array}$ & $\begin{array}{r}29,33 \quad 28,01 \\
\pm 0,85 \pm 0,85\end{array}$ \\
\hline $\begin{array}{l}\text { FOP24 carré } \\
\quad \text { (m Longissimus dorsi) }\end{array}$ & & & $\begin{array}{r}32,25 \\
\pm 1,64\end{array}$ & $\begin{array}{r}44,37 \\
\pm 1,54 \pm\end{array}$ & $\begin{array}{rr}28,39 & 40,90 \\
= & 1,07 \pm 1,01\end{array}$ & $\begin{array}{r}36,00 \quad 36,96 \\
\pm 0,85 \pm 0,86\end{array}$ \\
\hline $\begin{array}{l}\text { FOP24 jambon } 1 \\
\quad(\mathrm{~m} \text { Gracilis })\end{array}$ & & & $\begin{array}{r}31,76 \\
\pm 1,66\end{array}$ & $\begin{array}{r}35,78 \\
\pm 1,56 \pm\end{array}$ & $\begin{array}{r}30,36 \quad 35,36 \\
=1,08 \pm 1,02\end{array}$ & $\begin{array}{r}31,56 \quad 35,07 \\
\pm 0,86 \pm 0,87\end{array}$ \\
\hline $\begin{array}{l}\text { FOP24 jambon } 2 \\
\quad(\mathrm{~m} \text { Semi-membranosus })\end{array}$ & & & $\begin{array}{r}40,08 \\
\pm 1,70\end{array}$ & $\begin{array}{r}42,20 \\
\pm 1,60 \pm\end{array}$ & $\begin{array}{r}39,41 \quad 44,51 \\
=1,11 \pm 1,04\end{array}$ & $\begin{array}{r}40,93 \quad 42,17 \\
\pm 0,88 \pm 0,89\end{array}$ \\
\hline
\end{tabular}

Les effectifs varient de 78 à 346 selon la combinaison backcross-génotype. 
Table IV. Pourcentages de carcasses dans les classes commerciales AA + E + EE selon le génotype au locus $\mathrm{Hal}$, le sexe et le numéro de backcross.

\begin{tabular}{lcrccc}
\hline $\begin{array}{l}\text { Génotype au } \\
\text { locus Hal }\end{array}$ & Sexe & \multicolumn{3}{c}{$\begin{array}{c}\text { Backcross (\%) } \\
\text { 2 (7/8) }\end{array}$} & Piétrain pur (\%) \\
\hline \multirow{2}{*}{$N n$} & & $1(3 / 4)$ & (15/16) & \\
& Castrats & 3,52 & 3,76 & 12,71 & \\
& Femelles & 19,10 & 36,48 & 53,33 & \\
& Castrats & 20,24 & 22,34 & 44,26 & 44,96 \\
& Femelles & 64,21 & 73,71 & 83,67 & 88,20 \\
\hline
\end{tabular}

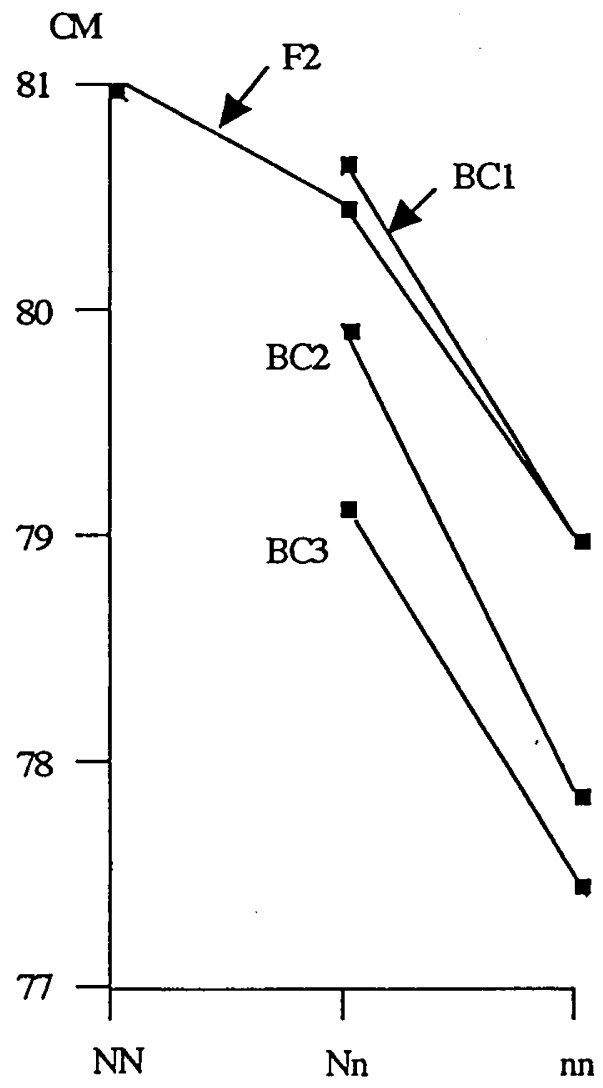

Fig 1. Longueur de carcasse en fonction du génotype au locus $\mathrm{Hal}$ et du niveau de reconstitution du génome Piétrain (F2 : $1 / 2 ; \mathrm{BC} 1: 3 / 4 ; \mathrm{BC} 2: 7 / 8 ; \mathrm{BC} 3: 15 / 16)$.

\section{$\%$}

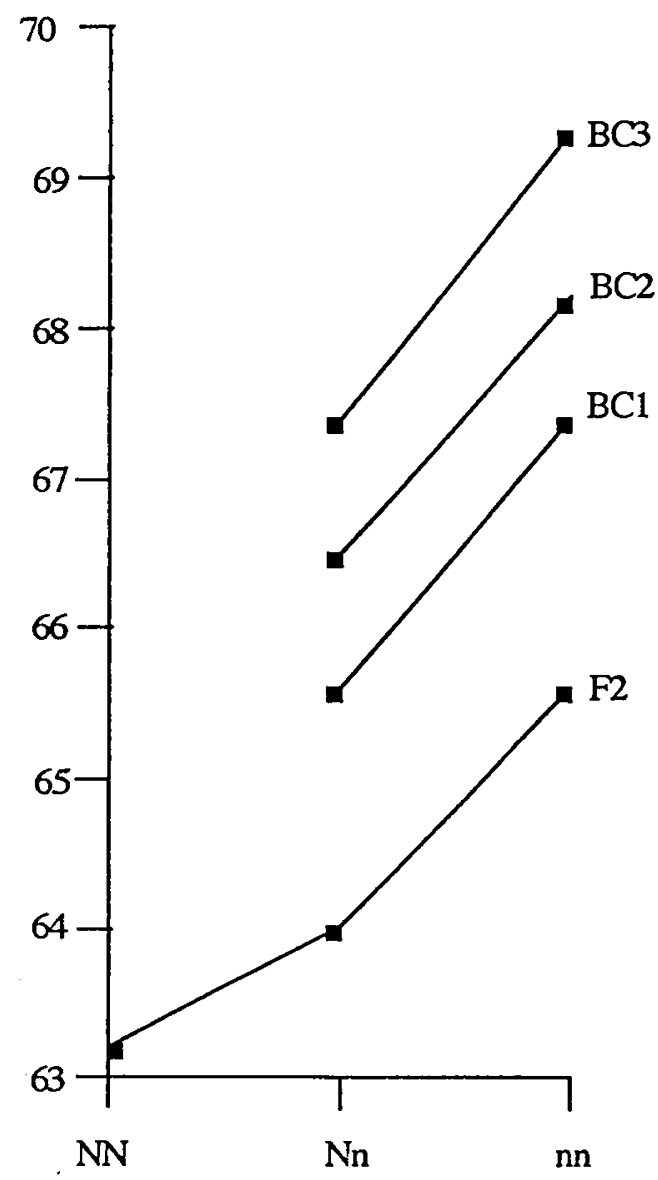

Fig 2. Proportion de morceaux maigres en fonction du génotype au locus $\mathrm{Hal}$ et du niveau de reconstitution du génome Piétrain (F2 : 1/2; BC1 : 3/4; BC2 : 7/8; BC3 : 15/16.) 
Castrats

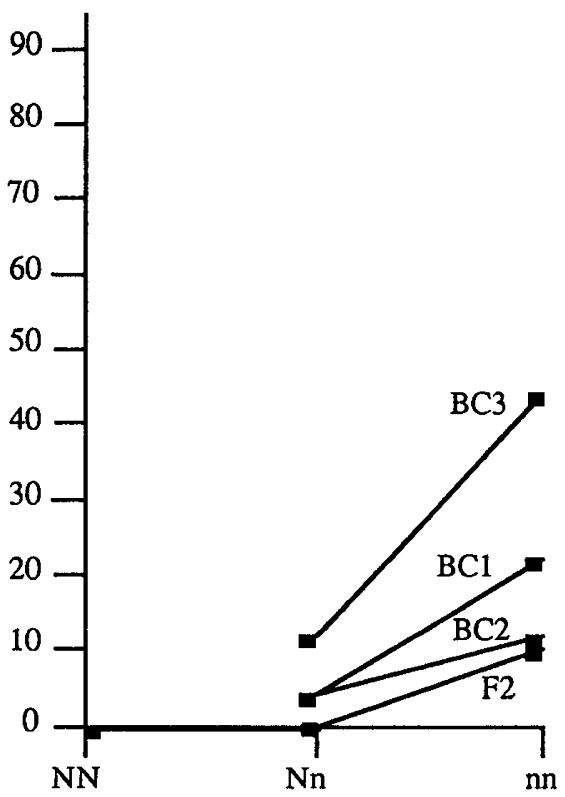

Femelles

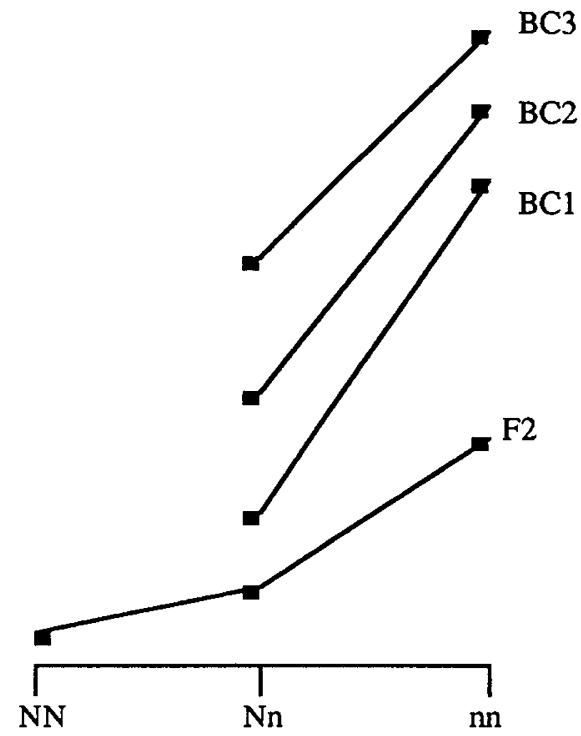

Fig 3. Pourcentage de carcasses dans les classes commerciales (AA $+E+E E)$ par sexe, en fonction du génotype au locus $\mathrm{Hal}$ et du niveau de reconstitution du génome Piétrain (F2: $1 / 2 ; \mathrm{BC} 1: 3 / 4 ; \mathrm{BC} 2: 7 / 8 ; \mathrm{BC} 3: 15 / 16)$.

\section{CONCLUSION}

Dans cette expérience de reconstitution du génome Piétrain autour de l'hétérozygotie au locus $\mathrm{Hal}$, la réponse de caractères tels que la longueur et la composition de la carcasse doit nécessairement présenter un caractère dégressif. En effet, s'il existe une différence de $4 \%$ pour le pourcentage de morceaux maigres entre l'animal $n n$ de la F2 et le Piétrain pur, on prévoit, en cas d'hérédité additive et en l'absence de sélection, le rythme suivant d'épuisement de la différence $=+2 \%$ (au backcross 1 , stade $3 / 4$ ) $;+1 \%$ (au backcross 2 , stade $7 / 8$ ) $;+0,5 \%$ (au backcross 3 , stade $15 / 16$ ); $+0,25 \%$ (backcross 4 , stade $31 / 32$ ), etc. La sélection des plus musclées parmi les femelles $N n$ destinées à produire le backcross suivant devait accélérer le processus. En fait, les sujets $n n$ au stade $15 / 16$, avec un pourcentage de morceaux maigres de $69,25 \%$, ne se distinguent plus des Piétrain purs chez lesquels ce pourcentage est de $69,3 \%$. Le programme prévoit d'aller jusqu'au stade $31 / 32$ avant que ne soit entrepris l'intercross, ce qui doit avoir pour effet de consolider l'acquis génétique et de raccourcir davantage la longueur du segment chromosomique d'origine Large White contenant le gène $N$. Une caractéristique essentielle de cette expérience est précisément ce retour permanent au Piétrain pur pour y puiser tous les gènes favorables au développement musculaire, en dehors du gène $n$. L'hétérozygote $N n$, au stade 15/16, a déjà un phénotype très acceptable même pour un marché qui, comme le marché belge, met tellement l'accent sur la conformation. Le contenu en 
morceaux maigres et l'indice de compacité 2 sont équivalents à ceux des porcs $n n$ du backcross 1 (stade $3 / 4$ ), avec toutefois cet avantage que ces porcs hétérozygotes $N n$ sont plus résistants au stress et présentent des critères de qualité de viande plus favorables.

En outre, on peut prévoir pour la viande fournie par l'hétérozygote une meilleure couleur, une teneur en pigments plus élevée, un meilleur pouvoir de rétention d'eau (Zhang et al, 1992; Klont et al, 1993). Il en est de même pour la teneur plus élevée en graisse intramusculaire, facteur favorable au persillé et aux qualités gustatives (Zhang et al, 1992). Bref, en enlevant 1 des 2 allèles $n$ mais en conservant tous les autres gènes, on corrige, partiellement tout au moins, les défauts que l'on peut rencontrer chez des Piétrain de conformation extrême.

L'opinion quant au sort à réserver au gène $n$ est moins négative depuis la découverte d'un test génomique d'application universelle et depuis qu'on reconnaît le profit pouvant résulter de l'exploitation du gène $n$ en dose simple. Celle-ci peut se concevoir de différentes manières :

- i) le croisement de femelles Piétrain $N N$, aboutissement du schéma ci-dessus, avec des verrats Piétrain classiques;

- ii) la poursuite indéfinie de croisements de retour au Piétrain pur avec truies Piétrain $N n$, ce qui assure une liaison constante avec la race pure;

- iii) le verrat Piétrain $N n$, alternative au verrat croisé Piétrain, utilisé en croisement terminal.

\section{REMERCIEMENTS}

Les auteurs remercient pour leur précieuse collaboration M M Bustin, le $\mathrm{D}^{\mathrm{r}} \mathrm{S}$ Dufrasne, l'Ir R Farnir, M ${ }^{\text {elle }} \mathrm{N}$ Brunetta.

Ils remercient également les 2 lecteurs anonymes pour leurs très utiles suggestions.

Ce travail a été réalisé sous les auspices de l'Institut pour l'encouragement de la recherche scientifique dans l'industrie et l'agriculture (IRSIA).

\section{RÉFÉRENCES}

Barton-Gade P (1981) The measurement of meat quality in pigs post-mortem. In : Porcine Stress and Meat Quality (T Froysten, E Slinde, N Standal, eds), Agric Food Res Soc As Norway, 205-218

Eikelenboom G, Nanni Costa L (1988) Fibre-optic probe measurements in Landrace pigs of different halothane phenotypes. Meat Sci, 23, 9-19

Eikelenboom G, Minkema D, Van Eldik P, Sybesma W (1978) Production characteristics of Dutch Landrace and Dutch Yorkshire pigs as related to their susceptibility for the halothane-induced malignant hyperthermia syndrome. Livestock Prod Sci 5, 277-284

Fuji J, Otsu K, Zorzato F, Deleon S, Khanna VK, Weiler JE, O'Brien PJ, MacLennan DH (1991) Identification of a mutation in porcine ryanodine receptor associated with malignant hyperthermia. Science $253,448-451$

Grobet L (1993) Diagnostics génomiques chez les animaux domestiques. Thèse, université de Liège.

Grobet L, Hanset R, Dasnois C (1992) Réponse au test à l'halothane et génotype au locus RYR1 du récepteur à la ryanodine chez des porcs croisés Piétrain. Ann Méd Vét 136, 249-257 
Hanset R, Dasnois C, Scalais S, Michaux C, Grobet L (1995) Génotypes au locus de sensibilité à l'halothane et caractères de croissance et de carcasse dans une F2 Piétrain $\times$ Large White. Genet Sel Evol 27, 63-76

Jensen $P$ (1981) Carcass and meat quality of pigs with known genotypes for halothane susceptibility. In : Porcine Stress and Meat Quality (T Froystein, E Slinde, N Standal, eds), Agric Food Res Soc As Norway, 267-273

Jensen P, Barton-Gade PA (1985) Performance and carcass characteristics of pigs with known genotypes for halothane susceptibility. In: Stress Susceptibility and Meat Quality in Pigs (JE Ludvingsen, eds), EAAP Publication $\mathrm{n}^{\circ} 33,80-87$

Klont RE, Lambooy E, Van Logtestijn JC (1993) Effect of preslaughter anesthesia on muscle metabolism and meat quality of pigs of different halothane genotypes. J Anim Sci 71, 1477-1485

Lampo P (1981) La sensibilité au stress chez le Landrace Belge. La relation entre le test d'anesthésie à l'halothane, les caractéristiques d'engraissement et de la carcasse. Revue de l'Agriculture 34, 213-220

Monin G, Sellier P, Ollivier L, Goutefongea R, Girard JP (1980) Carcass characteristics and meat quality of halothane-negative and halothane-positive Piétrain pigs. Meat Sci $5,413-423$

SAS User's Guide : Statistics (1985) SAS Inst Inc, CARY, NC, États-Unis ( $5^{\mathrm{e}}$ éd)

Simpson SP, Webb AJ (1989) Growth and carcass performance of British Landrace pigs heterozygous at the halothane locus. Anim Prod 49, 503-509

Wittmann W, Peschke W, Littmann E, Behringer J, Birkenmaier S, Dove P, Förster M (1993) Mast- und Schlachtleistungen von DL-Kastraten in Abhängigkeit von MHSgenotyp. Züchtungskunde 65, 197-205

Zhang W, Kuhlers DL, Rempel WE (1992) Halothane gene and swine performance. J Anim Sci $70,1307-1313$ 